\title{
STUDY OF MONTHLY SUPPLIER EVALUATION SCORECARD PARAMETERS OF INDIAN CAR MANUFACTURING INDUSTRY
}

\author{
Anand Patil \\ Research Scholar, Organizational Management, BRACTs Vishwakarma Institute of \\ Management, Savitribai Phule Pune University, Pune. Maharashtra, India

\section{Dr. Jyoti Gogte} \\ Research Guide, Organizational Management, BRACTs Vishwakarma Institute of \\ Management, Savitribai Phule Pune University, Pune. Maharashtra, India
}

\begin{abstract}
Growing automobile industry, make in India initiatives from government of India and increasing challenges of competitiveness has made supplier selection and periodic evaluation process more complex. In this paper, focus is given on periodic supplier evaluation scorecard parameters. The perceptions of experts from Indian car manufacturing industry are studied to understand their views on these parameters. Also studied the impact of variables like respondent's age, qualification, experience, department, designation, geographical location (region) and type of organization (OEM/Supplier) on these parameters. This study will be helpful to car manufacturing industry in India to understand the perceptions within industry about monthly supplier evaluation scorecard parameters. This in turn will help in improving monthly supplier evaluation scorecard systems and supplier performance.
\end{abstract}

Keywords: Monthly Supplier Evaluation Scorecard, Performance Measurements, Vendor Management, Supplier Evaluation, Supplier Performance.

Cite this Article: Anand Patil and Jyoti Gogte, Study of Monthly Supplier Evaluation Scorecard Parameters of Indian Car Manufacturing Industry, Journal of Management (JOM), 06 (3), 2019, pp. 42-50.

http://www.iaeme.com/JOM/issues.asp?JType=JOM\&VType=06\&IType=3

\section{INTRODUCTION}

Research work on supplier selection \& evaluation parameters starting from 23 parameters identified by Dickson's in 1966 is continuing over years. These parameters are changing over years, number of parameters getting added \& deleted every year. Their importance ranking also changing. Research work in the past is mainly focused on supplier selection scorecard parameters and less focus was given for periodic supplier evaluation scorecard parameters. 
Periodic evaluation process of suppliers is also very important considering retaining the supplier standard and improving it further. This is required to keep the supplier base competent and ready to face any challenges of car manufacturing Industry. Strong supplier base is key to success of any organization and gives an edge over competition. Indian car manufacturing industry has improved a lot over years still there are challenges particularly on various levels of suppliers (tier 1,2 ..). In India, each car manufacturing company is having its own supplier evaluation scorecard and process of evaluation on monthly basis. The parameters selected by these companies are varying from company to company. Attempt made in this research to understand perceptions of car manufacturing companies and their suppliers about these parameters.

\section{LITERATURE REVIEW}

Research work in past was mostly focused on supplier selection scorecard parameters and not on periodic supplier evaluation scorecard parameters. Therefore, researcher has collected monthly supplier evaluation scorecards of following key car manufacturing companies in India. FIAT India Automobiles Pvt Ltd., Ford Motors India, Honda Cars India Ltd, Mahindra and Mahindra Limited, Maruti Suzuki India Limited, General motors India, Renault Nissan automotive India Pvt Ltd, TATA Motors Limited, BMW India, Toyota Kirloskar Motors Limited, Volkswagen India Private Limited and Hyundai Motors India Limited.

Based on this study \& discussion with experts in industry, researcher has selected 16 key parameters as mentioned in Table 4.1. These parameters are mostly used for monthly performance evaluation scorecards of suppliers by these companies.

\section{RESEARCH METHODOLOGY}

Scope: India car manufacturing industry (OEMs and their suppliers in India), Period: Oct 2015 to Dec 2018

Sampling method: Judgmental method of sampling used as required information available with qualified engineers having experience related to supplier evaluation.

Data Collection: (a) Primary data: collected from 40 respondents of car manufacturing companies and their suppliers in India. Questionnaire was having likert scale (1-9) and options of open-ended response was provided to questions as appropriate (b) Secondary Data: data collected through existing supplier quality assurance manuals, Monthly supplier quality evaluation scorecard reports.

Data Analysis: XLSTAT and Microsoft Office tools used for analysis. Data Reliability: Data found reliable as per Cronbach's alpha value $>0.7$, Normality tests: Shapiro-Wilk, AndersonDarling and Lilliefors found p-value (Two-tailed) is less than significance level for alpha i.e. 0.05 therefore distribution is considered not normal distribution and non parametric methods Mann Whitney and Kruskal-Wallis tests used for analysis of data. ANOVA: Results are then verified by ANOVA, Tukey's HSD, REGWQ, Dunnett's test and correlations established between variables. Rank Analysis: descriptive statistics: using median and Inter quadratral range, graphs, charts

\section{ANALYSIS \& FINDINGS}

\subsection{Periodic Supplier evaluation parameters.}

Based on rank analysis using descriptive statistics the perceptions of experts from car manufacturing industry in India about identified 16 monthly supplier evaluation scorecard parameters are summarized and tabulated in table1 below. 
Table 1: Descriptive statistics: monthly supplier evaluation scorecard parameters.

\begin{tabular}{|c|c|c|c|c|c|c|c|c|c|c|c|c|c|c|c|c|c|}
\hline \# & Statistic & 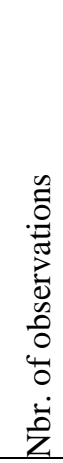 & 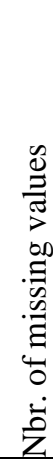 & $\underset{\Xi}{\Xi}$ & 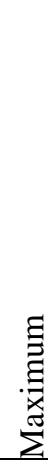 & 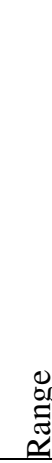 & 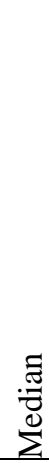 & $\sum_{\Sigma}^{\mathbb{E}}$ & 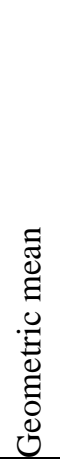 & 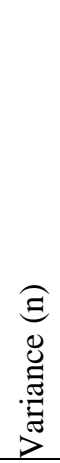 & 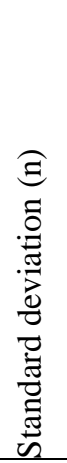 & 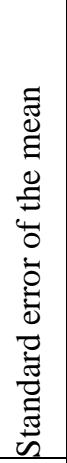 & 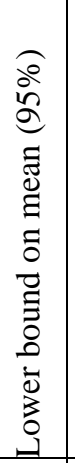 & 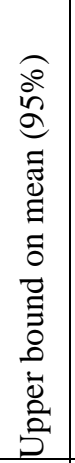 & 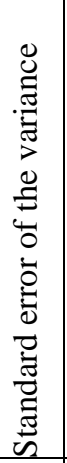 & 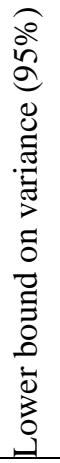 & 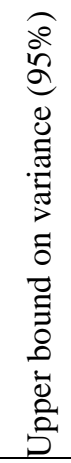 \\
\hline 1 & $\begin{array}{l}\text { Production } \\
\text { Line Stoppage } \\
\text { at customer } \\
\text { end } \\
\end{array}$ & 40 & 0 & 3 & 9 & 6 & 9 & $\begin{array}{l}\stackrel{8}{f} \\
\stackrel{+}{\infty} \\
\infty\end{array}$ & $\underset{\infty}{\infty}$ & 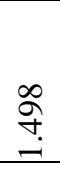 & 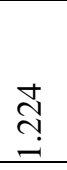 & $\frac{2}{2}$ & $\begin{array}{l}\stackrel{+}{0} \\
0 \\
\infty \\
\infty\end{array}$ & $\begin{array}{l}0 \\
+ \\
\infty \\
\infty \\
\infty\end{array}$ & 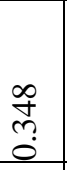 & $\bar{\sigma}$ & तె \\
\hline 2 & \begin{tabular}{|l|} 
Incoming \\
Quality \\
Rejection at \\
customer end
\end{tabular} & 40 & 0 & 3 & 9 & 6 & 9 & $\begin{array}{l}\text { \& } \\
\infty \\
\infty \\
\infty\end{array}$ & $\stackrel{\tilde{n}}{\infty}$ & $\stackrel{?}{\stackrel{2}{2}}$ & $\stackrel{\infty}{\circ}$ & 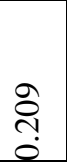 & $\begin{array}{l}0 \\
\infty \\
\infty \\
\end{array}$ & $\underset{\infty}{\stackrel{+}{\mathbb{N}}}$ & के & $\stackrel{\Xi}{\Xi}$ & $\begin{array}{l}\widetilde{N} \\
\infty \\
i \\
\text { i }\end{array}$ \\
\hline 3 & \begin{tabular}{|l} 
Production \\
Line \\
Rejections at \\
customer end \\
\end{tabular} & 40 & 0 & 1 & 9 & 8 & 9 & $\begin{array}{l}n \\
5 \\
\infty \\
\infty\end{array}$ & $\underset{\substack{\infty\\
}}{ }$ & $\vec{a}$ & $\stackrel{8}{\stackrel{2}{?}}$ & 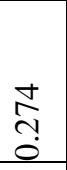 & $\begin{array}{l}\text { הั } \\
\text { ñ }\end{array}$ & 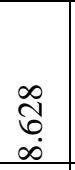 & 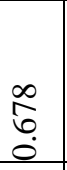 & $\begin{array}{l}8 \\
\dot{0} \\
i \\
i\end{array}$ & $\hat{\sigma}$ \\
\hline 4 & \begin{tabular}{|l|} 
Average \\
Response in \\
Resolving \\
Customer \\
Complaint \\
\end{tabular} & 40 & 0 & 4 & 9 & 5 & 9 & $\frac{i}{n}$ & $\begin{array}{l}\stackrel{n}{\infty} \\
\infty \\
\end{array}$ & $\stackrel{\infty}{\stackrel{\infty}{\stackrel{r}{i}}}$ & $\underset{\vec{J}}{\vec{Z}}$ & 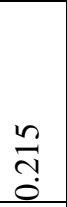 & $\frac{6}{n}$ & $\begin{array}{l}\mathbf{D} \\
\infty \\
\infty \\
\infty \\
\infty\end{array}$ & $\underset{\Xi}{ \pm}$ & $\stackrel{\overbrace{}}{\text { }}$ & 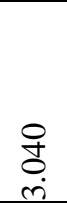 \\
\hline 5 & \begin{tabular}{|l|} 
Complaints \\
between \\
Production to \\
Sales (0 km \\
complaints) at \\
customer end \\
\end{tabular} & 40 & 0 & 1 & 9 & 8 & 9 & 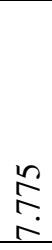 & $\underset{\text { oे }}{0}$ & $\underset{⿱ ㇒}{\stackrel{D}{n}}$ & 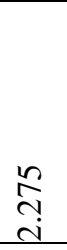 & 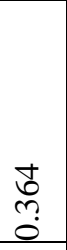 & 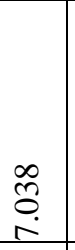 & $\begin{array}{l}1 \\
n \\
\infty \\
\infty\end{array}$ & ָָ & $\begin{array}{l}\vec{D} \\
n \\
n\end{array}$ & 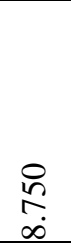 \\
\hline 6 & $\begin{array}{l}\text { After Sales } \\
\text { (Warranty) } \\
\text { Complaints at } \\
\text { customer end }\end{array}$ & 40 & 0 & 1 & 9 & 8 & 9 & $\stackrel{2}{N}$ & 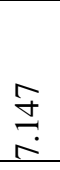 & $\underset{\stackrel{\leftrightarrow}{+}}{\stackrel{q}{+}}$ & $\begin{array}{l}\vec{\delta} \\
\stackrel{i}{i}\end{array}$ & 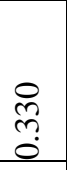 & $\frac{\tilde{n}}{r}$ & 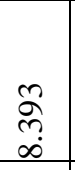 & \begin{tabular}{l}
$\hat{\infty}$ \\
\multirow{0}{0}{}
\end{tabular} & ふૂ & $\underset{\infty}{\stackrel{\infty}{\pi}}$ \\
\hline 7 & $\begin{array}{l}\text { Delivered Part } \\
\text { Timeline vs } \\
\text { Scheduled } \\
\text { Part Timeline } \\
\end{array}$ & 40 & 0 & 4 & 9 & 5 & 8 & ลิ & $\bar{f}$ & $\sqrt{1}$ & $\begin{array}{l}\infty \\
\\
\end{array}$ & \begin{tabular}{l}
$n$ \\
\multirow{2}{n}{} \\
0
\end{tabular} & 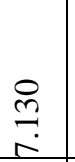 & $\underset{\infty}{\stackrel{D}{1}}$ & 先 & $\stackrel{5}{8}$ & \& \\
\hline 8 & $\begin{array}{l}\text { Default in } \\
\text { Supplies }\end{array}$ & 40 & 0 & 3 & 9 & 6 & 8 & $\stackrel{n}{n}$ & $\begin{array}{l}\stackrel{8}{0} \\
\text { ㄱ. }\end{array}$ & $\dot{m}$ & $\stackrel{\substack{n\\
}}{-}$ & 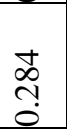 & ڤે & $\frac{8}{\infty}$ & $\begin{array}{c}\vec{m} \\
\stackrel{0}{o}\end{array}$ & $\frac{\widehat{b}}{\vec{i}}$ & $\begin{array}{l}\text { ते } \\
\text { nె: }\end{array}$ \\
\hline
\end{tabular}



Industry

\begin{tabular}{|c|c|c|c|c|c|c|c|c|c|c|c|c|c|c|c|c|c|}
\hline \# & Statistic & 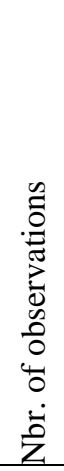 & 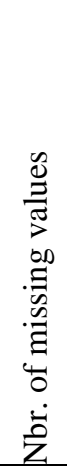 & 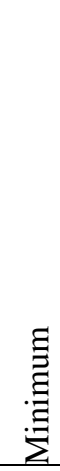 & 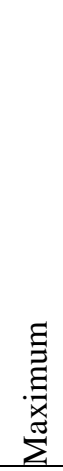 & 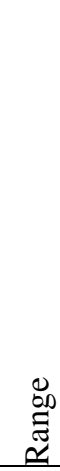 & 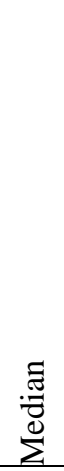 & $\sum_{\Sigma}^{\Xi}$ & 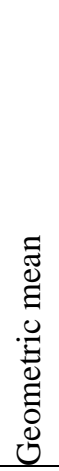 & 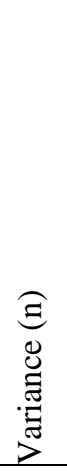 & 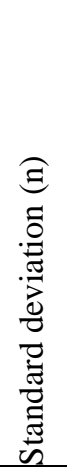 & 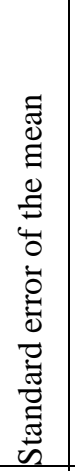 & 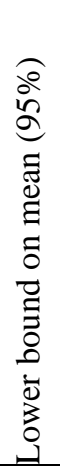 & 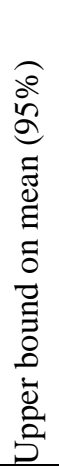 & 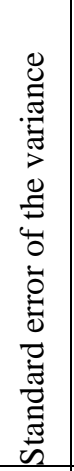 & 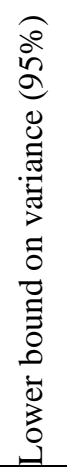 & 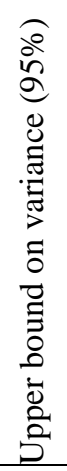 \\
\hline 9 & $\begin{array}{l}\text { Delivered Part } \\
\text { Quantity vs } \\
\text { Scheduled } \\
\text { Part Quantity }\end{array}$ & 40 & 0 & 1 & 9 & 8 & 8 & 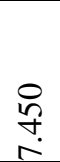 & 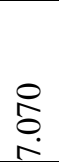 & $\stackrel{\infty}{\stackrel{\infty}{m}}$ & $\stackrel{\infty}{\stackrel{\infty}{\stackrel{\infty}{+}}}$ & $\begin{array}{l}0 \\
0 \\
1 \\
0\end{array}$ & $\underset{\substack{1 \\
0 \\
0}}{ }$ & 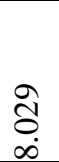 & $\underset{\stackrel{2}{+}}{\dot{0}}$ & $\begin{array}{c}\overrightarrow{\mathrm{N}} \\
\mathrm{i}\end{array}$ & 官 \\
\hline $\begin{array}{l}1 \\
0\end{array}$ & \begin{tabular}{|l} 
Product \\
Recalls from \\
Market
\end{tabular} & 40 & 1 & 1 & 9 & 8 & 8 & $\begin{array}{l}\stackrel{n}{\infty} \\
\stackrel{m}{r}\end{array}$ & مֶ. & 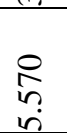 & $\begin{array}{l}\stackrel{8}{0} \\
\text { in } \\
\text { in }\end{array}$ & $\begin{array}{l}n \\
\infty \\
\infty \\
0 \\
0\end{array}$ & $\begin{array}{l}0 \\
\stackrel{0}{0}\end{array}$ & $\underset{\infty}{\stackrel{8}{\infty}}$ & $\bar{m}$ & $\frac{\infty}{\infty}$ & $\stackrel{\text { ă }}{a}$ \\
\hline $\begin{array}{l}1 \\
1\end{array}$ & $\begin{array}{l}\text { Controlled } \\
\text { Shipment } \\
\text { Levels }\end{array}$ & 40 & 0 & 1 & 9 & 8 & 7 & $\underset{\sim}{8}$ & 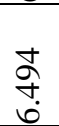 & 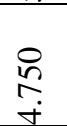 & $\stackrel{\curvearrowright}{\stackrel{2}{i}}$ & 守 & 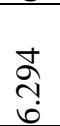 & 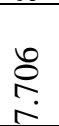 & $\underset{0}{0}$ & $\begin{array}{l}\stackrel{o}{े} \\
\stackrel{m}{m}\end{array}$ & ָै \\
\hline $\begin{array}{l}1 \\
2\end{array}$ & \begin{tabular}{|l} 
New Project \\
Development \\
Time
\end{tabular} & 40 & 0 & 1 & 9 & 8 & 7 & $\begin{array}{l}8 \\
\infty \\
0 \\
0\end{array}$ & $\underset{\square}{ \pm}$ & $\begin{array}{l}\stackrel{8}{\circ} \\
\stackrel{+}{+}\end{array}$ & ત્ત & $\begin{array}{c}n \\
\tilde{n} \\
0\end{array}$ & $\stackrel{\hat{a}}{\text {. }}$ & $\overrightarrow{\widetilde{n}}$ & $\stackrel{2}{\beth}$ & $\underset{d}{\vec{J}}$ & $\begin{array}{l}\infty \\
\infty \\
\infty \\
\infty\end{array}$ \\
\hline $\begin{array}{l}1 \\
3\end{array}$ & \begin{tabular}{|l} 
Returned \\
Shipments \\
(due to wrong \\
or excess \\
supplies) \\
\end{tabular} & 40 & 0 & 1 & 9 & 8 & $\begin{array}{l}6 . \\
5\end{array}$ & 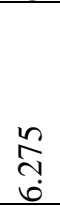 & $\begin{array}{l}0 \\
6 \\
\dot{0} \\
\dot{n}\end{array}$ & $\begin{array}{l}\text { oे } \\
\text { in }\end{array}$ & $\begin{array}{l}\hat{\infty} \\
\tilde{c}_{i}\end{array}$ & $\begin{array}{c}0 \\
\infty \\
ల \\
0\end{array}$ & 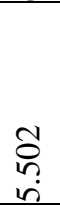 & $\underset{\sim}{\infty}$ & $\stackrel{\Xi}{\sim}$ & $\begin{array}{c}\tilde{\Omega} \\
\text { ळे }\end{array}$ & 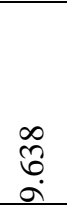 \\
\hline $\begin{array}{l}1 \\
4\end{array}$ & $\begin{array}{l}\text { Premium } \\
\text { Freights } \\
\text { (additional } \\
\text { cost for } \\
\text { express } \\
\text { shipment } \\
\text { during } \\
\text { urgency) } \\
\end{array}$ & 40 & 1 & 1 & 9 & 8 & 7 & 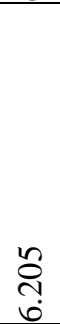 & $\begin{array}{l}\text { ڤે } \\
\text { ñ. }\end{array}$ & $\frac{\infty}{\stackrel{\infty}{r}}$ & $\begin{array}{l}\vec{\infty} \\
\stackrel{\infty}{c} \\
i\end{array}$ & 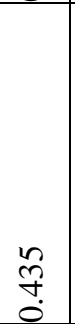 & $\begin{array}{l}\text { ते } \\
\text { in } \\
\text { in }\end{array}$ & 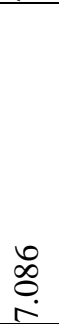 & $\underset{\hat{\sigma}}{0}$ & 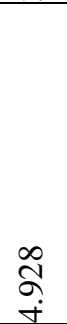 & 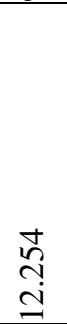 \\
\hline $\begin{array}{l}1 \\
5\end{array}$ & 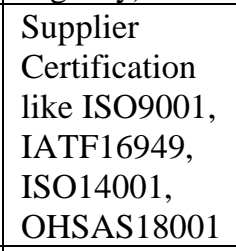 & 40 & 0 & 1 & 9 & 8 & $\begin{array}{l}6 . \\
5\end{array}$ & $\begin{array}{l}\hat{n} \\
\text { s. } \\
\end{array}$ & 㝘 & $\underset{b}{\stackrel{t}{*}}$ & $\begin{array}{l}\text { Ô} \\
\stackrel{0}{0} \\
\text { i }\end{array}$ & $\stackrel{5}{\ni}$ & $\frac{\varpi}{n}$ & $\begin{array}{l}\infty \\
\infty \\
0 \\
0\end{array}$ & $\stackrel{n}{n}$ & $\begin{array}{l}\widetilde{O} \\
\stackrel{0}{+} \\
+\end{array}$ & 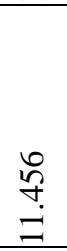 \\
\hline $\begin{array}{l}1 \\
6\end{array}$ & $\begin{array}{l}\text { Manufacturin } \\
\text { g Timeline or } \\
\text { Lead time }\end{array}$ & 40 & 1 & 1 & 9 & 8 & 6 & $\begin{array}{l}\vec{N} \\
\infty \\
\dot{n} \\
\dot{n}\end{array}$ & $\stackrel{\widehat{\widehat{a}}}{\vec{n}}$ & 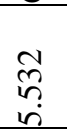 & $\begin{array}{l}\tilde{n} \\
\tilde{i} \\
i\end{array}$ & 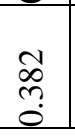 & $\begin{array}{l}\infty \\
0 \\
\dot{0} \\
\end{array}$ & مَ & $\begin{array}{c}\Omega \\
\text { లి } \\
\end{array}$ & 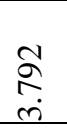 & ণ্ণি \\
\hline
\end{tabular}

\subsection{Corelations between variables:}

Corelations between monthly supplier performance evaluation scorecard parameters and variables - respondent's region (geographical location), type of organization (OEM/Supplier), department, designation, qualification, experience, age verified using Kruskal-Wallis test. Out of 16 parameters only three parameters have shown the correlations. These are shown in table 2 
Table 2: Kruskal-Wallis test - monthly supplier performance evaluation scorecard parameters

\begin{tabular}{|c|c|c|c|c|c|c|c|}
\hline VariablelTest & Region & $\begin{array}{l}\text { Org, } \\
\text { Type }\end{array}$ & Dept. & Desg. & Qual. & Exp. & Age \\
\hline $\begin{array}{l}\text { Incoming Quality Rejection at } \\
\text { customer end }\end{array}$ & 0.274 & 0.184 & 0.004 & 0.591 & 0.113 & 0.278 & 0.124 \\
\hline $\begin{array}{l}\text { Production Line Stoppage at } \\
\text { customer end }\end{array}$ & 0.050 & 0.594 & 0.296 & 0.158 & 0.127 & 0.442 & 0.498 \\
\hline $\begin{array}{l}\text { Average Response in Resolving } \\
\text { Customer Complaint }\end{array}$ & 0.904 & 0.618 & 0.109 & 0.500 & 0.018 & 0.235 & 0.894 \\
\hline
\end{tabular}

These corelations are then cross verified using ANOVA tests as shown in table 3 below

Table 3: ANOVA tests - monthly supplier performance evaluation scorecard parameters

\begin{tabular}{|c|c|c|c|c|c|c|c|c|c|}
\hline 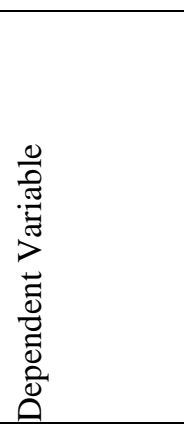 & 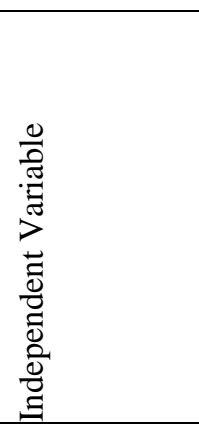 & 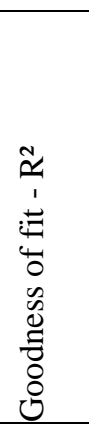 & 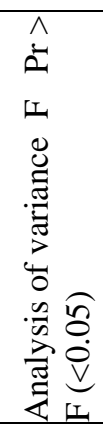 & 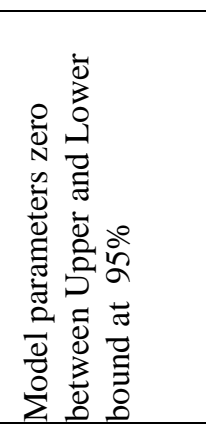 & 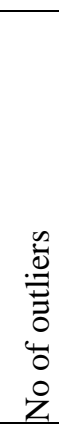 & 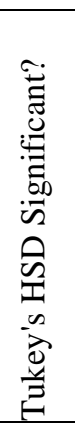 & 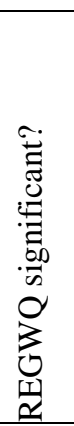 & 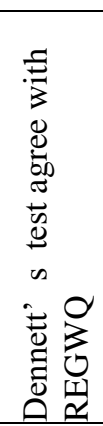 & 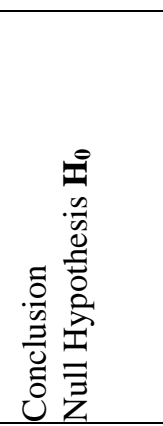 \\
\hline $\begin{array}{l}\text { Incoming } \\
\text { Quality } \\
\text { Rejection at } \\
\text { customer } \\
\text { end }\end{array}$ & Department & 0.356 & 0.018 & $\begin{array}{l}\text { Central } \\
\text { Quality-No } \\
\text { RestAll-Yes }\end{array}$ & 2 & No & No & Yes & $\begin{array}{l}\text { Accepted } \\
\text { No } \\
\text { corelation }\end{array}$ \\
\hline $\begin{array}{l}\text { Production } \\
\text { Line } \\
\text { Stoppage at } \\
\text { customer } \\
\text { end }\end{array}$ & Region & 0.245 & 0.006 & $\begin{array}{l}\text { South - Yes } \\
\text { North-No }\end{array}$ & 3 & Yes & Yes & Yes & $\begin{array}{l}\text { Rejected } \\
\text { Corelation } \\
\text { exists }\end{array}$ \\
\hline $\begin{array}{l}\text { Average } \\
\text { Response in } \\
\text { Resolving } \\
\text { Customer } \\
\text { Complaint }\end{array}$ & Qualification & 0.223 & 0.009 & $\begin{array}{l}\text { Graduate-No } \\
\text { Others-Yes }\end{array}$ & 1 & Yes & Yes & Yes & $\begin{array}{l}\text { Rejected } \\
\text { Corelation } \\
\text { exists }\end{array}$ \\
\hline
\end{tabular}

All the tests performed accepted existence of two out of three correlations mentioned above, those are - production line stoppage at customer end vs region (geographical location) and Average Response time in Resolving Customer Complaint vs qualification. Third correlation incoming quality rejection at customer end vs department which was identified by KruskalWallis test is rejected by Tukey's HSD and REGWQ tests which is also supported by Dennett's test indication no correlation or very weak correlation. These correlations are explained as below -

\subsubsection{Incoming Quality Rejection at customer end should be part of Monthly Supplier Evaluation vs Department}

From goodness of fit coefficient (determinant coefficient) $\mathrm{R}^{2}=0.356$ which indicates $35.6 \%$ of the variability explained. The other $64.4 \%$ are hidden in other variables which are not available, and which the model hides in "random errors". 

Industry

The analysis of variance: The test used here is the Fisher's F test. Given that the probability corresponding to the $\mathrm{F}$ value, in this case, is 0.018 , it means that we would take a $1.8 \%$ risk to conclude that the null hypothesis (no effect of the variable department on parameter incoming quality rejection at customer end) is wrong. So we can conclude with confidence that there is an effect of the region on the parameter incoming quality rejection at customer end.

Based on model parameters departments- customer quality, overall quality, plant quality, plant operations purchasing has an effect which $95 \%$ confidence range includes 0 , indicating that there is no evidence that these departments are very different from supplier quality. It seems here that there are two strong outliers $\left(20^{\text {th }} \& 23^{\text {rd }}\right.$ observations $)$ with a standard residual equal to $-3.714 \&-3.357$ respectively

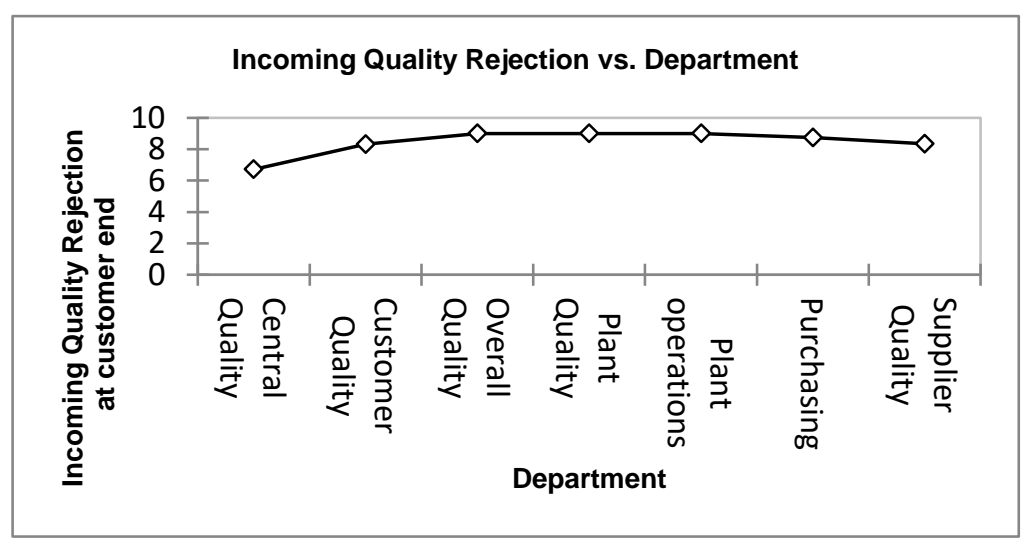

Figure 1 Incoming Quality Rejection vs. Department

Here no significant difference of opinion in any department except department "Cental Quality" which gives lesser weightage to parameter "Incoming quality rejection at customer end should be part of monthly supplier evaluation scoreacrd

Respondents from Overall Quality, Plant Quality and Plant Operations has given extreme importance with LS mean at 9.000. Respondents from departments Purchasing, Supplier Quality and Customer Quality has given slighly less importance with LS means at 8.750, 8.357 and 8.333 respoctively. Respondents from Central Quality departments has given lowest score with LS mean at 6.714 (ref Fig.1).

\subsubsection{Production Line Stoppage at customer end vs region}

From goodness of fit coefficient (determinant coefficient) $\mathrm{R}^{2}=0.245$ which indicates $24.5 \%$ of the variability explained. The other $75.5 \%$ are hidden in other variables which are not available, and which the model hides in "random errors".

The analysis of variance: The test used here is the Fisher's F test. Given that the probability corresponding to the $\mathrm{F}$ value, in this case, is 0.006 , it means that we would take a $0.6 \%$ risk to conclude that the null hypothesis (no effect of the region on parameter production line stoppage at customer end) is wrong.

So we can conclude with confidence that there is an effect of the region on the parameter production line stoppage at customer end. Note that the $\mathrm{R}^{2}$ is not very good $(0.245)$, meaning that some of the information offering a complementary explanation of the variations of the whiteness

From model parameters it is found that the region south has an effect which $95 \%$ confidence range includes 0 , indicating that there is no evidence that region south is very different from region west 
All values outside this interval are potential outliers or might suggest that the normality assumption is wrong. It seems here that there are two strong outliers $\left(11^{\text {th }} \& 22^{\text {nd }}\right.$ observations $)$ with a standard residual equal to $-2.818 \&-3.750$ respectively.

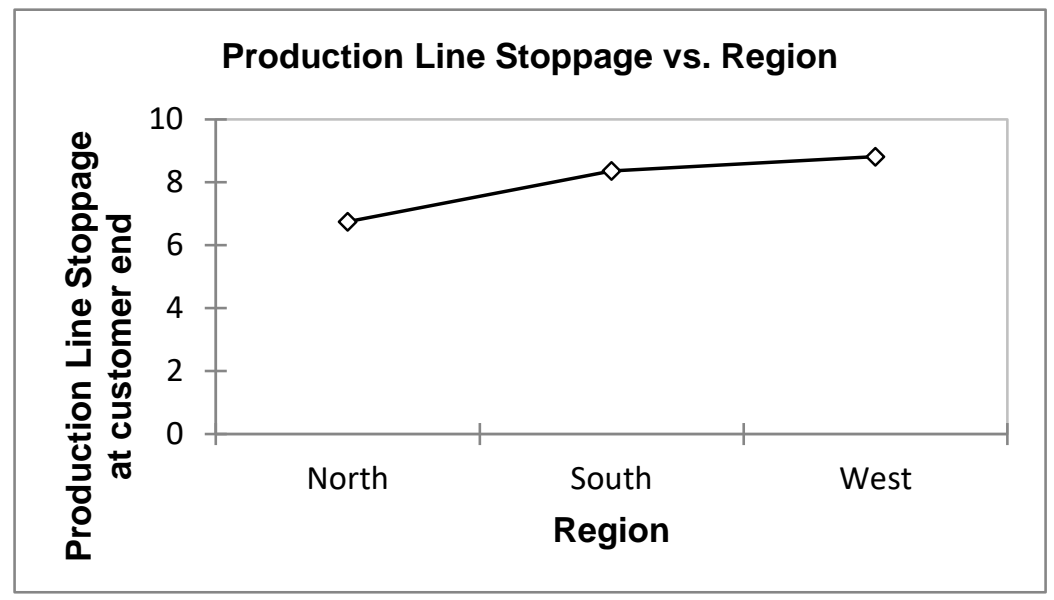

Figure 2 Production Line Stoppage vs. Region

From this we can say that region south and west do not have significant difference of opinion on parameter production line stoppage at customer end, both of these region give high priority to this parameter with LS means at $8.357 \& 8.818$ respectively while region north somewhat less priority to this parameter "production line stoppage at customer end should be part of monthly supplier evaluation scorecard parameter" with LS mean at 6.750 (ref Fig.2).

\subsubsection{Average Response in Resolving Customer Complaint vs Qualification:}

From goodness of fit coefficient (determinant coefficient) $\mathrm{R}^{2}=0.223$ which indicates $22.3 \%$ of the variability explained. The other $76.7 \%$ are hidden in other variables which are not available, and which the model hides in "random errors".

The analysis of variance: The test used here is the Fisher's F test. Given that the probability corresponding to the $\mathrm{F}$ value, in this case, is 0.009 , it means that we would take a $0.9 \%$ risk to conclude that the null hypothesis (no effect of the region on parameter production line stoppage at customer end) is wrong.

So we can conclude with confidence that there is an effect of the region on the parameter production line stoppage at customer end. Note that the $\mathrm{R}^{2}$ is not very good (0.223), meaning that some of the information offering a complementary explanation of the variations of the whiteness is missing, which is no real surprise.

From model parameters we can say that the qualification diploma has an effect which $95 \%$ confidence range includes 0 , indicating that there is no evidence that qualification diploma is very different from qualification post graduate.

All values outside this interval are potential outliers or might suggest that the normality assumption is wrong. It seems here that there is one strong outlier $\left(36^{\text {th }}, 37^{\text {th }} \& 39^{\text {th }}\right.$ observation $)$ with a standard residual equal to $-3.133,2.400 \& 2.133$ respectively. 


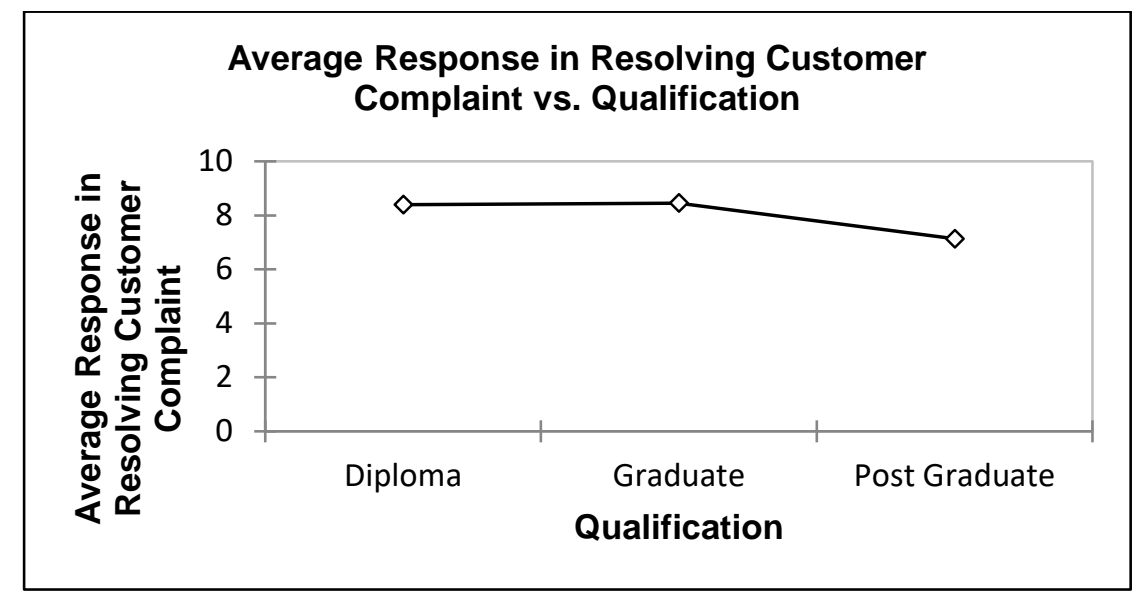

Figure 3 Average Response in Resolving Customer Complaint vs. Qualification

Respondents with Qualification Diploma and Graduates has given nearly equal importance at higher side with LS means at 8.400 and 8.450 respoctively. Respondents with Qualification Post Graduate has given comparitively less importance with LS mean at 7.133 (ref Fig.3).

\section{CONCLUSIONS}

Apart from supplier selection, Indian car manufacturing industry has their unique monthly supplier evaluation scorecard system. Each car manufacturing company has its own monthly supplier evaluation scorecards whose parameters are varying from company to company.

There were three correlation observed between these parameters and respondent's variables i.e. (1) Incoming Quality Rejection at customer end vs department (2) Production Line Stoppage at customer end vs region (geographical location) (3) Average Response in Resolving Customer Complaint vs qualification. No influence observed on these parameters by respondent's age, designation, experience and organization type (OEM/supplier)

There is lot of difference of opinion among the respondents on these parameters as range varying between 5 to 8 on 1-9 likert scale for all 16 parameters.

\section{FUTURE SCOPE}

This research can be extended to other industries in India and/or International studies can be performed. Difference of opinions on these parameters among car manufacturing industry in India can be further explored. Study can also be done to understand if there are any factors that has influence on supplier performance evaluation scorecard. Scope can be extended to other parameters than the 16 parameters covered in this study.

\section{REFERENCES}

[1] S. Hossein Cheragh, Mohammad Dadashzadeh, Muthu (2011), "Subramanian, Critical Success Factors For Supplier Selection: An Update", Journal of Applied Business Research Volume 20, Number 2011

[2] Sagar, M. K., \& Singh, D. (2012). "Supplier Selection Criteria : Study of Automobile Sector in India", International Journal of Engineering Research and Development e-ISSN: 2278-067X, p-ISSN: 2278-800X, www.ijerd.com Volume 4, Issue 4 (October 2012), PP. 34-39

[3] Macedonia, E. V. N., \& Skopje, A. D. (2013). "Key Performance Criteria For Vendor Selection - A Literature Review", Management Research And Practice Vol. 5 Issue 2 (2013) pp: 63-75 
[4] Patil, A. N. (2014). "Modern Evolution in Supplier Selection Criteria and methods". International Journal of Management Research \& Review, 4(5), 616-623.

[5] Susanty, A., Puspitasari, D., \& Marga, B. V. (2014). "Using Scorecard to Measure Supply Chain Performance" SMEs Hand-Stamped Batik, 12(2), 78-90.

[6] Bigliardi B., Bottani E. (2014). "Supply Chain Performance Measurement: A Literature review and Pilot study among Italian Manufacturing Companies". International Journal of Engineering, Science and Technology, Vol. 6 No. 3, pp. 1-16

[7] Article, I. R., Felice, F. De, Deldoost, M. H., Faizollahi, M., \& Petrillo, A. (2015). "Performance Measurement Model for the Supplier Selection Based on AHP Invited" Review Article. http://doi.org/10.5772/61702

[8] Shpend Imeri, Khuram Shahzad, Josu Takala, Yang Liu, Ilkka Sillanpa ä, Tahir Ali (2015) "Evaluation and Selection Process of Suppliers Through Analytical Framework: An Empirical Evidence of Evaluation Tool", Management and Production Engineering Review, Volume 6, Number 3, September 2015, pp. 10-20

[9] Fabio De Felice1, Mostafa H. Deldoost2, Mohsen Faizollahi3 and Antonella Petrillo, (2015), "Performance Measurement Model for the Supplier Selection Based on AHP", International Journal of Engineering Business Management, 2015, 7:17

[10] Zeljko Sevic,(2017), "Criteria for supplier selection: A literature review", International Journal of Engineering, Business and Enterprise Applications, 19(1), December 2016February 2017, pp. 23-27

[11] Ehsan Khan Mohammadi, Hamid Reza Talaie, Hossein Safari \& Reza Salehzadeh (2018), "Supplier evaluation and selection for sustainable supply chain management under uncertainty Conditions", International Journal of Sustainable Engineering · January 2018

[12] Monthly supplier evaluation scorecards of following key car manufacturing companies in India (1) FIAT India Automobiles Pvt Ltd., (2) Ford Motors India, (3) Honda Cars India Ltd, (4) Mahindra and Mahindra Limited, (5) Maruti Suzuki India Limited, (6) General motors India, (7) Renault Nissan automotive India Pvt Ltd, (8) TATA Motors Limited, (9) BMW India, (10) Toyota Kirloskar Motors Limited, (11) Volkswagen India Private Limited and (12) Hyundai Motors India Limited. 